\title{
Cilostazol on the expression of ICAM-1, VCAM-1 and inflammatory factors in plasma in patients with thromboangiitis obliterans
}

\author{
FUCHEN SONG ${ }^{1}$, BO JI ${ }^{1}$ and TING $\mathrm{CHEN}^{2}$ \\ Departments of ${ }^{1}$ Vascular Surgery and ${ }^{2}$ Ophtalmology, Affiliated Hospital of Shandong University of \\ Traditional Chinese Medicine, Jinan, Shandong 250011, P.R. China
}

Received December 14, 2017; Accepted June 28, 2018

DOI: $10.3892 / \mathrm{etm} .2018 .6436$

\begin{abstract}
The effects of cilostazol on the expression of intercellular adhesion molecule-1 (ICAM-1), vascular cell adhesion molecule-1 (VCAM-1) and inflammatory factors in plasma in patients with thromboangiitis obliterans (TAO) were studied. Plasma viscosity, fibrinogen, total cholesterol (TC) and triglyceride (TG) were detected for the healthy control, TAO and cilostazol groups, respectively. Results showed that compared with those in the control group, the plasma viscosity, fibrinogen, TC and TG levels in TAO group were significantly increased. By contrast, compared with those in TAO group, the plasma viscosity, fibrinogen, TC and TG levels in the cilostazol group were significantly decreased. ELISA results revealed that ICAM-1 and VCAM-1 expression levels in TAO group were obviously increased compared with those in control group. ICAM-1 and VCAM-1 expression levels in cilostazol group were obviously decreased compared with those in TAO group. According to RT-PCR, the mRNA expression levels of IL-1 $\beta$, IL- 6 and TNF- $\alpha$ in TAO group were significantly higher than those in control group, while the levels in cilostazol group were significantly decreased compared with those in TAO group. In addition, RT-PCR and western blotting proved that expression of both mRNA and protein of ICAM-1 and VCAM-1 in TAO group was significantly increased and obviously decreased after administration of cilostazol. The results of analysis of variance showed that the differences of ICAM-1 and VCAM-1 expression was statistically significant among the control, TAO and cilostazol groups $(\mathrm{p}<0.01)$. Cilostazol can significantly reduce the TAO-induced abnormal increase in ICAM-1, VCAM-1 and inflammatory factor expression in
\end{abstract}

Correspondence to: Dr Bo Ji, Department of Vascular Surgery, Affiliated Hospital of Shandong University of Traditional Chinese Medicine, 16369 Jingshi Road, Jinan, Shandong 250011, P.R. China E-mail: gqhee891@163.com

Key words: cilostazol, inflammatory factors, thromboangiitis obliterans, ICAM-1, VCAM-1 plasma in patients. It was proven that cilostazol has a good anti-TAO effect.

\section{Introduction}

Thromboangiitis obliterans (TAO) is a common peripheral vascular disease, which is endarteritis jointly caused by $\mathrm{T}$ cell-mediated cellular immunity and B cell-mediated humoral immunity $(1,2)$. The vascular endothelial cell functions of TAO patients are severely damaged, among which the expression of intercellular adhesion molecule-1 (ICAM-1) and vascular cell adhesion molecule-1 (VCAM-1) are increased in thickened vascular endothelial cells and some inflammatory cells, seriously harming life and health $(3,4)$. Cilostazol is a phosphodiesterase inhibitor, which inhibits cyclic adenosine monophosphate (cAMP) degradation and transformation by inhibiting the cAMP phosphodiesterase activity in platelets and smooth muscle cells, ultimately leading to the increased cAMP level in platelets and blood vessels (5). Cilostazol has significant effects of dilating blood vessels and inhibiting platelet aggregation, but its role in TAO has not been reported yet.

In the present study, the effects of cilostazol on the expression of ICAM-1, VCAM-1 and inflammatory factors in plasma in TAO patients were investigated to clarify the roles of cilostazol in the occurrence and development of TAO.

\section{Patients and methods}

Patients. Patients in the TAO and cilostazol groups were diagnosed with TAO in the Affiliated Hospital of Shandong University of Traditional Chinese Medicine (Jinan, China). It was confirmed by color Doppler flow imaging instrument that all patients suffered from peripheral arterial ischemia to varying degrees. In the TAO group, there were 23 patients, including 13 males and 10 females, aged 34-55 years. In the cilostazol group, there were 20 patients, 12 males and 8 females, aged 32-56 years, who were treated with administration of cilostazol (50 mg/time, twice a day). In the control group, there were 22 healthy subjects, including 12 males and 10 females, aged 35-50 years; patients with hypertension, hyperlipidemia or cardiovascular and cerebrovascular organic 
Table I. Primer sequences in RT-PCR.

\begin{tabular}{lll}
\hline Genes & \multicolumn{1}{c}{ Forward primer (5'-3') } & \multicolumn{1}{c}{ Reverse primer (5'-3') } \\
\hline Human $\beta$-actin & GAGCCGGGAAATCGTGCGT & GGAAGGAAGGCTGGAAGATG \\
Human IL-1 $\beta$ & CTGAGCACCTTCTTTCCCTTCA & TGGACCAGACATCACCAAGCT \\
Human IL-6 & TGGCTGAAAAAGATGGATGCT & TCTGCACAGCTCTGGCTTGT \\
Human TNF- $\alpha$ & TGTAGCCCATGTTGTAGCAAACC & GAGGACCTGGGAGTAGATGAGGTA
\end{tabular}

diseases were eliminated. There were no significant differences in the sample size, sex and the age of subjects among the three groups. The study was approved by the Ethics Committee of Affiliated Hospital of Shandong University of Traditional Chinese Medicine (Shandong, China). Signed written informed consents were obtained from the patients and/or guardians.

Main reagents. Enzyme-linked immunosorbent assay (ELISA) kits were obtained from R\&D Systems (Minneapolis, MN, USA); the bicinchoninic acid (BCA) protein quantification kit was purchased from Beyotime Institute of Biotechnology (Shanghai, China); TRIzol total RNA extraction kits were obtained from Tiangen Biotech, Co., Ltd., (Beijing, China); reverse transcription-polymerase chain reaction (RT-PCR) kits were purchased from Tiangen Biotech; glyceraldehyde-3-phosphate dehydrogenase (GAPDH), as well as ICAM-1 and VCAM-1 monoclonal antibodies were obtained from Cell Signaling Technology (Danvers, MA, USA).

\section{Experimental methods}

Blood routine examinations. Fasting elbow venous blood (4 ml) was drawn from all the subjects in the control, TAO and cilostazol groups in the early morning. Heparin sodium was used for anticoagulation of samples used in blood rheology examination; the plasma viscosity, fibrinogen, total cholesterol (TC) and triglyceride (TG) levels in each group were detected, respectively, and the differences among the three groups were compared.

Detection of ICAM-1 and VCAM-1 expression levels via ELISA. The ICAM-1 and VCAM-1 expression levels in the control, TAO and cilostazol groups were detected using the human ICAM-1 and VCAM-1 ELISA kits, and the expression differences were recorded.

RT-PCR analyses of inflammation-related factors. Appropriate number of tissues in the control, TAO and cilostazol groups were quickly transferred into $1 \mathrm{ml}$ TRIzol reagent for full tissue grinding to prodcue homogenate. After 5-min standing at room temperature, the sample was completely cleaved, followed by centrifugation at $12,000 \mathrm{x}$ g at $4^{\circ} \mathrm{C}$ for $5 \mathrm{~min}$. The supernatant was carefully removed, added with chloroform and mixed evenly, and after 5-min standing at room temperature, the mixture was centrifuged at $12,000 \mathrm{x} \mathrm{g}$ at $4^{\circ} \mathrm{C}$ for $15 \mathrm{~min}$. The supernatant was carefully taken and added with the same volume of isopropanol, and after 10-min standing at room temperature, the mixture was centrifuged at $12,000 \mathrm{x} \mathrm{g}$ at $4^{\circ} \mathrm{C}$ for $10 \mathrm{~min}$. The sediment was retained, added with $75 \%$ ethanol and mixed evenly to wash the RNA sediment. Finally,
RNase-free water was added to fully dissolve the sediment. The optical density $(\mathrm{OD})_{260} / \mathrm{OD}_{280}$ ratio and the RNA concentration were measured. The amplification was performed step by step based on the primer sequences shown in Table I according to the protocol, and the RT-PCR analyses were performed for reaction products.

RT-PCR analyses of ICAM-1 and VCAM-1. Tissues in the control, TAO and cilostazol groups were transferred into an Eppendorf (EP) tube containing RNAiso Plus extract. After 5 -min standing at room temperature, the sample was completely cleaved, followed by centrifugation at $12,000 \mathrm{xg}$ at $4^{\circ} \mathrm{C}$ for $5 \mathrm{~min}$. Then, the supernatant was added with $0.2 \mathrm{ml}$ chloroform and mixed evenly, and after 5-min standing at room temperature, the mixture was centrifuged at $12,000 \mathrm{x} \mathrm{g}$ at $4^{\circ} \mathrm{C}$ for $15 \mathrm{~min}$. The supernatant was added with the same volume of isopropanol, and after 10-min standing at room temperature, the mixture was centrifuged at $12,000 \mathrm{x}$ g at $4^{\circ} \mathrm{C}$ for $10 \mathrm{~min}$; the supernatant was removed carefully and the sediment was retained, added with $1 \mathrm{ml} \mathrm{75 \%}$ ethanol and mixed evenly, followed by centrifugation at $12,000 \mathrm{x}$ g at $4^{\circ} \mathrm{C}$ for $5 \mathrm{~min}$. Then the supernatant was removed carefully, and the above procedures were repeated once. After RNA sediment was washed, the solution was discarded, and RNase-free water was added. Part of total RNA solution was diluted into $1 \mu \mathrm{g} / \mu \mathrm{l}$, and the reverse transcription reaction solution was prepared according to instructions of PrimeScript ${ }^{\circledR}$ RT Reagent kit with gDNA Eraser, and the corresponding RNA samples were added for reverse transcription to obtain complementary DNA (cDNA). cDNA was stored at $-20^{\circ} \mathrm{C}$, and the mRNA level was detected according to the instructions of the SYBR $^{\circledast}$ Premix Ex Taq ${ }^{\mathrm{TM}}$ II (Tli RNaseH Plus) kit. Primer sequences used were: ICAM-1: 5'-3'AGGTGTGATATCCGG TAGAA; 3'-5' CCTTCTAAGTGGTTGGAACA; VCAM-1: 5'-3' TCTACGCTGACAATGAATCC; 3'-5' ACTTGACTG TGATCGGCTTC.

Western blot analysis of ICAM-1 and VCAM-1. Tissues in the control, TAO and cilostazol groups were taken and washed with ice cold normal saline. According to the instructions of the whole protein extraction kit, immunoprecipitation (IP) lysate containing phenylmethanesulfonyl fluoride (PMSF) and protease inhibitors was added and tissues were fully ground on ice. Then, the tissue homogenate was centrifuged at $12,000 \mathrm{xg}$ at $4^{\circ} \mathrm{C}$ for $10 \mathrm{~min}$; the supernatant was taken and centrifuged at $12,000 \mathrm{x} \mathrm{g}$ at $4^{\circ} \mathrm{C}$ for $20 \mathrm{~min}$, and the supernatant was collected again. After protein quantification according to the instructions of the protein kit, the protein sample containing the same amount of total protein was added into each well, 

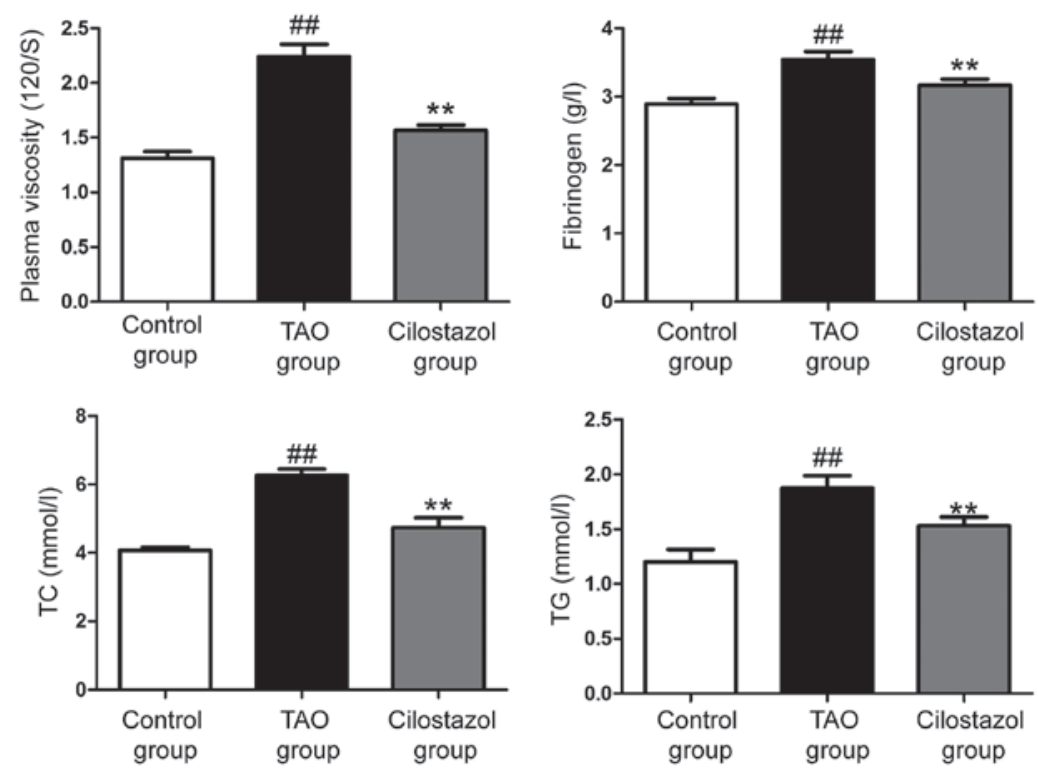

Figure 1. Routine blood examinations of control, TAO and cilostazol groups. Compared with the control group, ${ }^{\# \#}$ p $<0.01$; compared with the TAO group, *** $\mathrm{p}<0.01$.
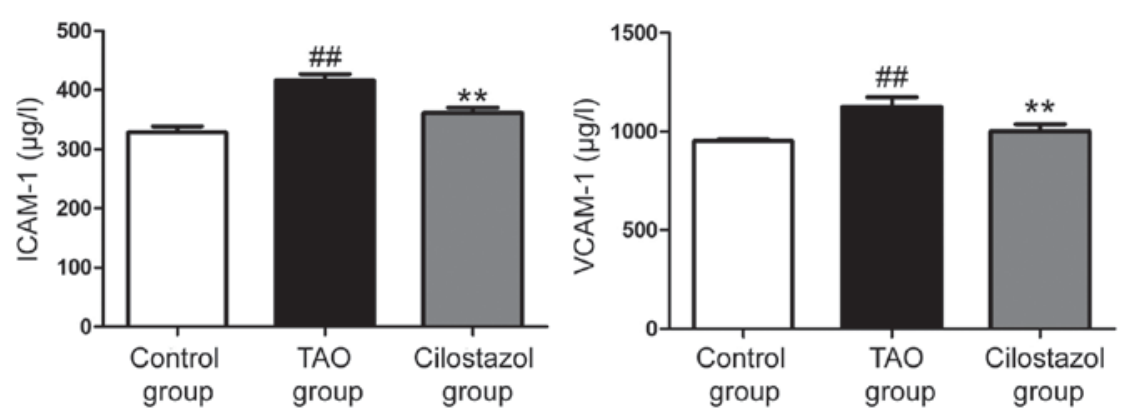

Figure 2. Detection of ICAM-1 and VCAM-1 expression in each group via ELISA. Compared with the control group, ${ }^{\#}$ p $<0.01$; compared with the TAO group, $*$ p $<0.01$.

followed by sodium dodecyl sulfate polyacrylamide gel electrophoresis (SDS-PAGE) under constant pressure of $220 \mathrm{~V}$, until the bromophenol blue reached the bottom of the gel. Based on the molecular weight of the target protein, the gel was cut and the isolated protein was transferred onto the polyvinylidene fluoride (PVDF) membrane. The protein-attached PVDF membrane was sealed in $5 \%$ skim milk powder at room temperature for $3 \mathrm{~h}$ on a shaker and incubated with the corresponding primary antibody $(1: 1,000)$ at $4^{\circ} \mathrm{C}$ overnight. The next day, the membrane was fully washed with TTBS (10 min/time, 3 times), incubated with the secondary antibody $(1: 2,000)$ at room temperature for $1 \mathrm{~h}$, and washed again with TTBS (10 min/time, 3 times), followed by color development using ECL developing solution and photography. Finally, the bands were analyzed and data were processed.

Statistical analysis. Experimental data are presented as mean \pm standard deviation (SD). Statistical Product and Service Solutions (SPSS) 17.0 software (SPSS, Inc., Chicago, IL, USA) was used for the statistical analysis of experimental results. The t-test was used for the comparison of means between two groups. One-way analysis of variance (ANOVA) followed by post hoc test (Least Significant Difference) was used for the comparison of means among groups. P-test was used for the pairwise comparison. $\mathrm{P}<0.05$ indicated the difference was statistically significant.

\section{Results}

Effects of cilostazol on TAO-induced abnormalities in routine blood tests. Compared with those in the control group, the plasma viscosity, fibrinogen, TC and TG levels in the TAO group were significantly increased. Compared with those in the TAO group, the plasma viscosity, fibrinogen, TC and TG levels in the cilostazol group were significantly decreased, suggesting that cilostazol can effectively affect the TAO-induced abnormalities in routine blood tests and improve TAO (Fig. 1).

Detection of ICAM-1 and VCAM-1 expression in each group via ELISA. The expression of ICAM-1 and VCAM-1 in the control, TAO and cilostazol groups were detected using the ELISA kit. The results showed that ICAM-1 and VCAM-1 expression levels in the TAO group were obviously increased compared with those in the control group. ICAM-1 and VCAM-1 expression levels in the cilostazol group were obviously decreased compared with those in the TAO group, 

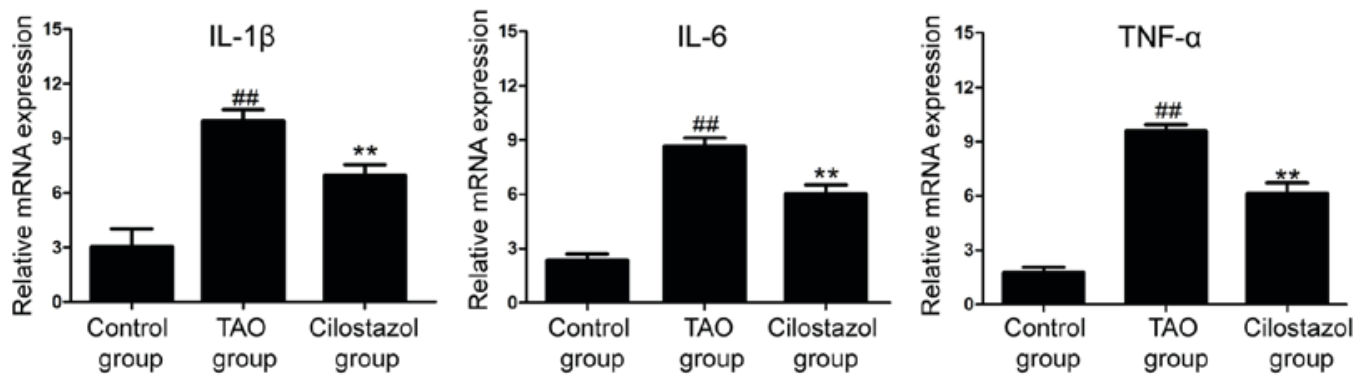

Figure 3. RT-PCR results of inflammation-related factors (IL-1 $\beta$, IL-6 and TNF- $\alpha$ ). Compared with the control group, ${ }^{\# \# ~}<<0.01$; compared with the TAO group, ** $\mathrm{p}<0.01$.
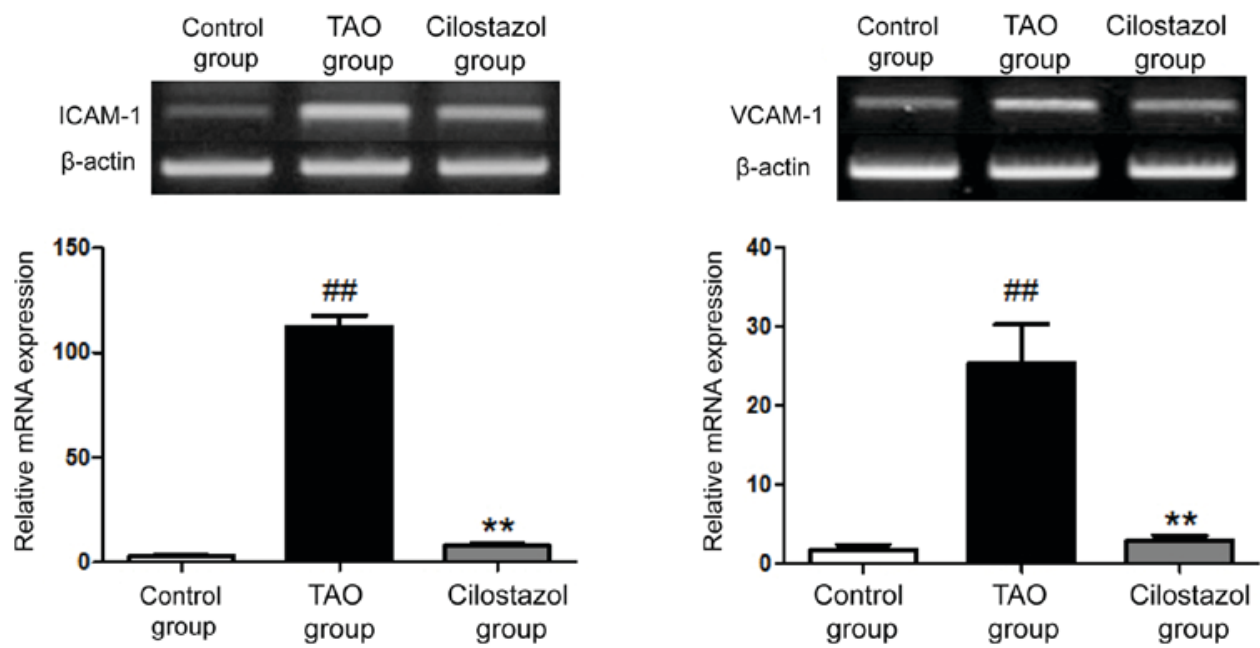

Figure 4. RT-PCR results of ICAM-1 and VCAM-1. Compared with the control group, ${ }^{\# \#} \mathrm{p}<0.01$; compared with the TAO group, ${ }^{* * *} \mathrm{p}<0.01$.
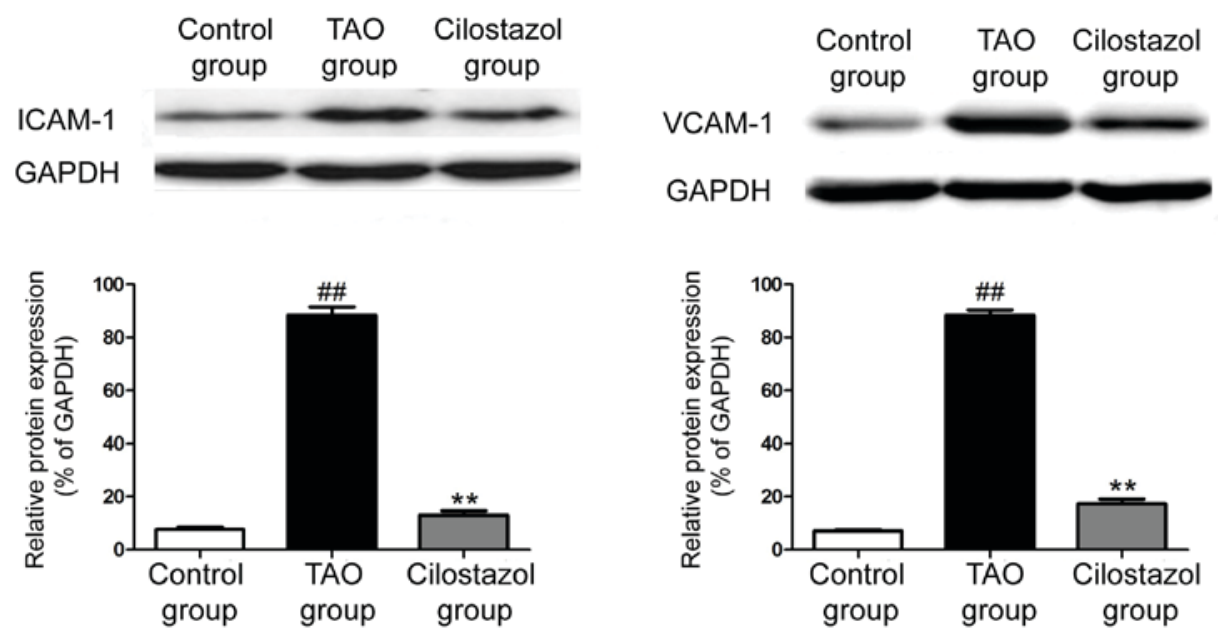

Figure 5. Western blot results of ICAM-1 and VCAM-1. Compared with the control group, ${ }^{\sharp \# \#}$ p $<0.01$; compared with the TAO group, ${ }^{* *} \mathrm{p}<0.01$.

indicating that cilostazol can reduce the TAO-induced abnormal expression of ICAM-1 and VCAM-1 (Fig. 2).

RT-PCR results of inflammation-related factors. Total RNA was extracted from the control group, the TAO group and the cilostazol group, and RT-PCR was performed. The results revealed that the mRNA expression levels of IL-1 $\beta$, IL- 6 and TNF- $\alpha$ in the TAO group were significantly higher than those in the control group, while the levels in the cilostazol group were significantly decreased compared with those in the TAO group (Fig. 3).

RT-PCR results of ICAM-1 and VCAM-1. RT-PCR showed that the mRNA expression of ICAM-1 and VCAM-1 in the TAO group was significantly increased, which was effectively reversed after administration of cilostazol (Fig. 4). 
Western blot results of ICAM-1 and VCAM-1. The protein expression of ICAM-1 and VCAM-1 in the control group, the TAO group and the cilostazol group were detected via western blotting. The results revealed that the protein expressions of ICAM-1 and VCAM-1 in the TAO group were significantly increased, which were obviously decreased after administration of cilostazol (Fig. 5).

\section{Discussion}

Thromboangiitis obliterans (TAO) is a disease with unknown cause and mechanism, mainly involving the middle and small arteries and veins in upper and lower limbs (6). TAO seriously endangers people's lives, therefore effective treatment for TAO has been investigated to clarify its molecular mechanism (7-9). Cilostazol, as a selective PDE-III inhibitor, is clinically widely used in the treatment of peripheral vascular diseases caused by metabolic syndrome and intermittent claudication $(10,11)$, which can significantly reduce the levels of soluble adhesion molecules (such as ICAM-1 and VCAM-1) in plasma in diabetic patients, thereby delaying the development of microvascular disease (12).

With the deepening of study using immune biochemical techniques, a variety of factors have been found to be involved in the occurrence and development of TAO, such as inflammation, apoptosis, oxidative stress, macrophages and lymphocytes (13-15). ICAM-1 is expressed on the vascular endothelium and binds to the ligand to promote the firm adhesion between monocytes and endothelial cells (16-18). Moreover, ICAM-1 is highly expressed in TAO, which is closely related to the TAO-induced increased expression of inflammatory factors, such as IL-1 $\beta$, IL- 6 and TNF- $\alpha$. In addition, VCAM-1 is expressed in vascular endothelial cells, which mediates the adhesion among lymphocytes, monocytes and endothelial cells and participates in many important pathophysiological processes, and it may be an important indicator for vascular dysfunction or vascular disease progression. It has been reported that the incidence of TAO is closely related to ICAM-1 and VCAM-1 (19,20).

In this study, routine blood examinations (plasma viscosity, fibrinogen, TC and TG levels) were performed for the control group, the TAO group and the cilostazol group, respectively. The routine blood examination results showed that compared with those in the control group, the plasma viscosity, fibrinogen, TC and TG levels in TAO group were significantly increased; compared with those in the TAO group, the plasma viscosity, fibrinogen, TC and TG levels in the cilostazol group were significantly decreased. Then the differences of ICAM-1 and VCAM-1 expression in the three groups were detected via ELISA. The results showed that ICAM-1 and VCAM-1 expression levels in the TAO group were obviously increased compared with those in the control group; ICAM-1 and VCAM-1 expression levels in the cilostazol group were obviously decreased compared with those in the TAO group. Besides, the mRNA expression of IL- $1 \beta$, IL- 6 and TNF- $\alpha$ in the three groups were detected via RT-PCR. The results revealed that the mRNA expression levels of IL- $1 \beta$, IL- 6 and TNF- $\alpha$ in the TAO group were significantly higher than those in the control group, while the levels in the cilostazol group were significantly decreased compared with those in the TAO group. The mRNA and protein expressions of ICAM-1 and VCAM-1 in the three groups were detected via RT-PCR and western blotting, respectively. It was found that both mRNA and protein expression of ICAM-1 and VCAM-1 in the TAO group were significantly increased, which were obviously decreased after administration of cilostazol. Moreover, the results of ANOVA showed that the differences of ICAM-1 and VCAM-1 expression were statistically significant among the control group, the TAO group and the cilostazol group.

In conclusion, cilostazol can significantly reduce the TAO-induced abnormal increase in ICAM-1, VCAM-1 and inflammatory factor expression in plasma of patients. It was proven that cilostazol has a good anti-TAO effect. We expect that cilostazol, as an anti-inflammatory drug, can provide a new, feasible and effective solution for the prevention and treatment of TAO in the future.

\section{Acknowledgements}

Not applicable.

\section{Funding}

No funding was received.

\section{Availability of data and materials}

All data generated or analyzed during this study are included in this published article.

\section{Authors' contributions}

FS and BJ designed the study. TC and FS collected the patient data. BJ and TC analyzed the patient data. All authors read and approved the final manuscript.

\section{Ethics approval and consent to participate}

The study was approved by the Ethics Committee of Affiliated Hospital of Shandong University of Traditional Chinese Medicine (Jinan, China). Signed written informed consents were obtained from the patients and/or guardians.

\section{Patient consent for publication}

Not applicable.

\section{Competing interests}

The authors declare no competing interests.

\section{References}

1. Wysokinski WE, Kwiatkowska W, Sapian-Raczkowska B, Czarnacki M, Doskocz R, Kowal-Gierczak B and Wysokinski WE: Sustained classic clinical spectrum of thromboangiitis obliterans (Buerger's disease). Angiology 51: 141-150, 2000.

2. Tse TS, Mcbane RD, Stanson AW, Ballman KV, Mikhail MA and Cooper LT: Secular trends and long-term survival in thromboangiitis obliterans. J Am Coll Cardiol 39: 265, 2002.

3. Sasaki S, Sakuma M and Yasuda K: Current status of thromboangiitis obliterans (Buerger's disease) in Japan. Int J Cardiol 75 (Suppl 1): S175-S181, 2000. 
4. Sun XL, Law BY, de Seabra Rodrigues Dias IR, Mok SWF, $\mathrm{He}$ YZ and Wong VK: Pathogenesis of thromboangiitis obliterans: Gene polymorphism and immunoregulation of human vascular endothelial cells. Atherosclerosis 265: 258-265, 2017.

5. Marchetti C, Poggi P, Cornaglia AI, Farina A and Rizzo S Morphologic characteristics of initial lymphatics of the healthy and diseased human gingiva. Anat Rec 255: 175-179, 1999.

6. Iwai T: Periodontal bacteremia and various vascular diseases. J Periodontal Res 44: 689-694, 2009.

7. Chen YW, Nagasawa T, Wara-Aswapati N, Ushida Y, Wang D, Takeuchi Y, Kobayashi H, Umeda M, Inoue Y, Iwai T, et al: Association between periodontitis and anti-cardiolipin antibodies in Buerger disease. J Clin Periodontol 36: 830-835, 2009.

8. Chen Z, Takahashi M, Naruse T, Nakajima T, Chen YW, Inoue Y, Ishikawa I, Iwai T and Kimura A: Synergistic contribution of CD14 and HLA loci in the susceptibility to Buerger disease. Hum Genet 122: 367-372, 2007.

9. Maslowski L, McBane R, Alexewicz P and Wysokinski WE: Antiphospholipid antibodies in thromboangiitis obliterans. Vasc Med 7: 259-264, 2002.

10. Undas A, Nowakowski T, Cieśla-Dul M and Sadowski J: Abnormal plasma fibrin clot characteristics are associated with worse clinical outcome in patients with peripheral arterial disease and thromboangiitis obliterans. Atherosclerosis 215: 481-486, 2011.

11. Zheng P, Chen SJ and Shao HZ: Studies on hypercoagulation state in thromboangiitis obliterans. Chin Med J (Engl) 102: 67-71, 1989.

12. Avcu F, Akar E, Demirkiliç U, Yilmaz E, Akar N and Yalçin A: The role of prothrombotic mutations in patients with Buerger's disease. Thromb Res 100: 143-147, 2000.

13. Brodmann M, Renner W, Stark G, Winkler M, Pabst E, Hofmann $\mathrm{C}$ and Pilger E: Prothrombotic risk factors in patients with thrombangitis obliterans. Thromb Res 99: 483-486, 2000.
14. Dellalibera-Joviliano R, Joviliano EE and Evora PR: Determination of kininogens levels and kallikrein/kininase II activities in patients with thromboangiitis obliterans. Scand J Immunol 72: 128-133, 2010.

15. Idei N, Nishioka K, Soga J, Hidaka T, Hata T, Fujii Y, Fujimura N, Maruhashi T, Mikami S, Teragawa H, et al: Vascular function and circulating progenitor cells in thromboangitis obliterans (Buerger's disease) and atherosclerosis obliterans. Hypertension 57: 70-78, 2011.

16. Azizi M, Boutouyrie P, Bura-Rivière A, Peyrard S, Laurent $S$ and Fiessinger JN: Thromboangiitis obliterans and endothelial function. Eur J Clin Invest 40: 518-526, 2010.

17. Halacheva K, Gulubova MV, Manolova I and Petkov D: Expression of ICAM-1, VCAM-1, E-selectin and TNF- $\alpha$ on the endothelium of femoral and iliac arteries in thromboangiitis obliterans. Acta Histochem 104: 177-184, 2002.

18. Luo Y, Feng J, Xu Q, Wang W and Wang X: NSun2 deficiency protects endothelium from inflammation via mRNA methylation of ICAM-1. Circ Res 118: 944-956, 2016.

19. Slavov ES, Stanilova SA, Petkov DP and Dobreva ZG: Cytokine production in thromboangiitis obliterans patients: New evidence for an immune-mediated inflammatory disorder. Clin Exp Rheumatol 23: 219-226, 2005.

20. Małecki R, Zdrojowy K and Adamiec R: Thromboangiitis obliterans in the 21st century: a new face of disease. Atherosclerosis 206: 328-334, 2009. International (CC BY-NC-ND 4.0) License. 